Pacific Journal of Mathematics

ON SPACES OF DISTRIBUTIONS STRONGLY REGULAR WITH 


\section{ON SPACES OF DISTRIBUTIONS STRONGLY REGULAR WITH RESPECT TO PARTIAL DIFFERENTIAL OPERATORS}

\section{Z. ZIELEZNY}

A distribution $T$ in $\Omega$ is said to be strongly regular with respect to the differential operator $P(D)$, if $P^{k}(D) T, k=$ $0,1, \cdots$, are of bounded order in any open set $\Omega^{\prime} \subset \subset \Omega$. Necessary and sufficient conditions on the polynomials $P$ and $Q$ are established in order that a distribution $T$ strongly regular with respect to $P(D)$ be strongly regular with respect to $Q(D)$.

Let $P(D)$ be a partial differential operator in $R^{n}$ with constant coefficients and $P^{k}(D), k=1,2, \cdots$, its successive iterations. The following result is due to L. Hörmander ([3], Theorem 3.6 and Remark on p. 233):

If $P(D)$ is hypoelliptic and $T$ is a distribution such that $P^{k}(D) T$, $k=1,2, \cdots$, have a bounded order in any relatively compact open subset of $R^{n}$, then $T$ is a $C^{\infty}$-function.

In other words, the space $\mathscr{E}_{P}$ of distributions in $R^{n}$ "strongly regular with respect to $P(D)$ " is contained in the space $\mathscr{E}$ of $C^{\infty}$ functions; in this case $\mathscr{E}_{P}=\mathscr{E}_{\text {. }}$ The concept of strong regularity with respect to $P(D)$ coincides with that of strong regularity in some variables (see [6], p. 453), when $P(D)$ is the Laplace operator in those variables.

Suppose now that given are two arbitrary partial differential operators $P(D)$ and $Q(D)$. Then the question arises: Under what conditions on $P$ and $Q$ is $\mathscr{E}_{P} \subset \mathscr{E}_{Q}$ ? In particular, if $P(D)$ is "Qhypoelliptic," i.e. all solutions $U \in \mathscr{D}^{\prime}$ of the equation

$$
P(D) U=0
$$

are in $\mathscr{E}_{Q}$, must then be $\mathscr{E}_{P} \subset \mathscr{E}_{Q}$ ? The $Q$-hypoelliptic operators were studied (in a slightly different but equivalent version) and characterized by E. A. Gorin and V. V. Grušin [2].

In this paper we give necessary and sufficient conditions for the inclusion $\mathscr{E}_{P}(\Omega) \subset \mathscr{E}_{Q}(\Omega)$, where $\mathscr{E}_{P}(\Omega)$ and $\mathscr{E}_{Q}(\Omega)$ are the spaces of "strongly regular" distributions on an arbitrary open set $\Omega \subset R^{n}$. These conditions are, in general, stronger than the $Q$-hypeollipticity of $P(D)$. If the inclusion in question holds for every $Q$-hypoelliptic operator $P(D)$, then $Q(D)$ must be hypoelliptic and the problem reduces to that in Hörmander's theorem stated above.

1. The spaces $\mathscr{E}_{P}(\Omega)$ and $C_{P}^{\prime \prime, \infty}(\Omega)$. 
Let $\Omega$ be a nonempty open subset of $R^{n}$. A distribution $T \in \mathscr{D}^{\prime}(\Omega)$ will be called strongly regular with respect to the differential operator $P(D)$, if to every open set $\Omega^{\prime}$ having compact closure contained in $\Omega$ (we express this by writing $\Omega^{\prime} \subset \subset \Omega$ ) there exists an integer $m \geqq 0$ such that $P^{k}(D) T, k=0,1, \cdots$, are all of order $\leqq m$ in $\Omega^{\prime}$, i.e. the restrictions of $P^{k}(D) T$ to $\Omega^{\prime}$ are all in $\mathscr{D}^{\prime m}\left(\Omega^{\prime}\right)^{1}$. We denote by $\mathscr{E}_{P}(\Omega)$ the space of all distributions in $\Omega$, which are strongly regular with respect to $P(D)$. We also denote by $C_{P}^{\mu, \infty}(\Omega)$, where $\mu$ is an integer $\geqq 0$, the space of all $C^{\mu}$-functions in $\Omega$ such that $P^{k}(D) D^{\alpha} f,|\alpha| \leqq \mu$, $k=0,1, \cdots$, are continuous functions; here $\alpha=\left(\alpha_{1}, \cdots, \alpha_{n}\right)$ and $|\alpha|=\alpha_{1}+\cdots+\alpha_{n}$.

Consider now the spaces $\mathscr{E}_{P}(\Omega)$ and $\mathscr{E}_{Q}(\Omega)$ corresponding to the differential operators $P(D)$ and $Q(D)$ respectively.

THEOREM 1. If $\mathscr{E}_{P}(\Omega) \subset \mathscr{E}_{Q}(\Omega)$, then to any open set $\Omega^{\prime} \subset \subset \Omega$ there exists an integer $\mu \geqq 0$ such that the restriction mapping $f \rightarrow$ $f \mid \Omega^{\prime}$ maps $C_{P}^{\prime \prime, \infty}(\Omega)$ into $C_{Q}^{0, \infty}\left(\Omega^{\prime}\right)$.

Proof. Let $\Omega^{\prime}$ be an open set satisfining the assumption $\Omega^{\prime} \subset \subset \Omega$. We first prove the existence of nonnegative integers $\nu$ and $m$ such that

$$
\left\{Q^{k}(D) f \mid \Omega^{\prime}: f \in C_{P}^{\nu, \infty}(\Omega), k=0,1, \cdots\right\} \subset \mathscr{D}^{\prime m}\left(\Omega^{\prime}\right) .
$$

Suppose that inclusion (1) does not hold for any $\nu$ and $m$. Then to every $\nu$ and $m$ there exist a function $f \in C_{P}^{\nu, \infty}(\Omega)$ and a $k$ such that $Q^{k}(D) f \mid \Omega^{\prime} \notin \mathscr{D}^{\prime m}\left(\Omega^{\prime}\right)$. Thus we can find strictly increasing sequences of positive integers $\nu_{i}, m_{i}$ and $k_{i}$, and a sequence of functions $f_{i}$ with the following properties:

$$
\begin{gathered}
f_{i} \in C_{P}^{y, i}, \infty(\Omega), \\
Q^{k}(D) f_{i} \mid \Omega^{\prime} \in \mathscr{D}^{\prime m_{i}}\left(\Omega^{\prime}\right), k=0,1, \cdots, \\
Q^{k_{i}}(D) f_{i} \mid \Omega^{\prime} \text { is of order } m_{i}, \\
q k_{i}<\nu_{i+1},
\end{gathered}
$$

where $i=1,2, \cdots$, and $q$ is the order of the operator $Q(D)$.

We denote by $\Omega_{i}, i=1,2, \cdots$, open subsets of $\Omega$ such that

$$
\Omega_{i} \subset \subset \Omega_{i+1} \text { and } \bigcup_{i=1}^{\infty} \Omega_{i}=\Omega .
$$

Next we set

$$
a_{1}=1 \text { and } a_{i}=2^{-i} M_{i}^{-1}, \quad i=2,3, \cdots,
$$

${ }_{1} P^{0}(D)$ is the identity operator, i.e. $P^{0}(D) T=P$. 
where

$$
M_{i}=\sup \left\{\left|P^{k}(D) f_{i}(x)\right|+\left|Q^{l}(D) f_{i}(x)\right|+1\right\}
$$

and the supremum is taken over all $x \in \Omega_{i}$ and $k, l=0,1, \cdots, k_{i-1}$. Note that $Q^{l}(D) f_{i}, l=0,1, \cdots, k_{i-1}$, are continuous functions in $\Omega$, because of (5).

The function

$$
f=\sum_{i=1}^{\infty} a_{i} f_{i}
$$

is defined and continuous in $\Omega$, since the $f_{i}^{\prime}$ 's are continuous in $\Omega$ and the series converges there almost uniformly. Moreover, for any $k$ we have (distributionally)

$$
P^{k}(D) f=\sum_{i=1}^{\infty} a_{i} P^{k}(D) f_{i} .
$$

But each term of the last series is a continuous function in $\Omega$, by (1). Also

$$
a_{i} \sup _{x \in \Omega_{j}}\left|P^{k}(D) f_{i}(x)\right| \leqq 2^{-i}
$$

whenever $k<i$ and $j \leqq i$, by the definition of $\alpha_{i}$. Hence it follows that the series (7) converges almost uniformly in $\Omega$, for any $k$. Consequently $f \in C_{P}^{0, \infty}(\Omega) \subset \mathscr{E}_{P}(\Omega)$.

We now show that $f$ is not in $\mathscr{E}_{Q}(\Omega)$, which is a contradiction to our hypothesis. We write

$$
g_{j}=\sum_{i=1}^{j} a_{i} f_{i} \text { and } h_{j}=\sum_{i=j+1}^{\infty} a_{i} f_{i} .
$$

In view of (3) and (4), the restriction of $Q^{k_{j}}(D) g_{j}$ to $Q^{\prime}$ is a distribution of order $m_{j}$. On the other hand, $Q^{k_{j}}(D) f_{i}, i=j+1, j+2, \cdots$, are continuous functions in $\Omega$, because of (2) and (5). Furthermore, by the definition of the $a_{i}$ 's, the series

$$
\sum_{i=j+1}^{\infty} a_{i} Q^{k_{J}}(D) f_{i}
$$

converges almost uniformly in $Q$, and so $Q^{k_{j}}(D) h_{j}$ is in $\Omega$ a continuous function. Thus

$$
Q^{k_{j}}(D) f=Q^{k_{j}}(D) g_{j}+Q^{k_{j}}(D) h_{j}
$$

is in $\Omega^{\prime}$ a distribution of order $m_{j}$. Since $m_{j} \rightarrow \infty, f$ is not in $\mathscr{E}_{Q}(\Omega)$. This contradiction proves (1).

Consider now the fundamental solution $E$ of the iterated Laplace equation, i.e. 


$$
\Delta^{\gamma} E=\delta
$$

For sufficiently large $\gamma, E$ is $m$ times continuously differentiable. Therefore every distribution $T$ on $\Omega^{\prime}$ such that $\Delta^{r} T \in \mathscr{D}^{\prime m}\left(\Omega^{\prime}\right)$ is, in fact, a continuous function (see [5], vol. 2, p. 47). We choose $\mu=$ $2 \gamma+\nu$, where $\nu$ is the integer occurring in (1). Then, if $f \in C_{P}^{\mu, \infty}(\Omega)$, it follows that $\Delta^{\gamma} f \in C_{P}^{\nu, \infty}(\Omega)$ whence, in view of $(1), Q^{k}(D) \Delta^{\gamma} f \mid \Omega^{\prime}=$ $\Delta^{r} Q^{k}(D) f \mid \Omega^{\prime} \in \mathscr{D}^{\prime m}\left(\Omega^{\prime}\right)$. Thus, by what we said before, $Q^{k}(D) f \mid \Omega^{\prime}$ is a continuous function, for every $k=0,1, \cdots$, i.e. $f \mid Q^{\prime} \in C_{Q}^{0, \infty}\left(\Omega^{\prime}\right)$. The proof is complete.

2. Necessary conditions. We proceed to derive necessary conditions for the inclusion $\mathscr{E}_{P}(\Omega) \subset \mathscr{E}_{Q}(\Omega)$. In view of Theorem 1 it suffices to find necessary conditions for the inclusion

$$
\left\{f \mid \Omega^{\prime}: f \in C_{P}^{\mu, \infty}(\Omega)\right\} \subset C_{Q}^{0, \infty}\left(\Omega^{\prime}\right) .
$$

We accomplish this by means of the standard argument based on the closed graph theorem and the Seidenberg-Tarski theorem (see [1]).

Let $\Omega_{j}, j=1,2, \cdots$, be open sets satisfying conditions (6). We define the topology in $C_{P}^{\mu, \infty}(\Omega)$ by means of the semi-norms

$$
v_{j}(f)=\sup \left|P^{k}(D) D^{\alpha} f(x)\right|,
$$

where the supremum is taken over all $x \in \Omega_{j},|\alpha| \leqq \mu$ and $k \leqq j$. Similarly, if $\Omega_{j}^{\prime}, j=1,2, \cdots$, are open sets satisfying conditions analogous to (6) with $\Omega$ replaced by $\Omega^{\prime}$, we define the topology in $C_{Q}^{0, \infty}\left(\Omega^{\prime}\right)$ by means of the semi-norms

$$
w_{j}(f)=\sup _{x \in Q^{\prime}, k \leqq j}\left|Q^{k}(D) f(x)\right| .
$$

Then $C_{P}^{\mu, \infty}(\Omega)$ and $C_{Q}^{0, \infty}\left(\Omega^{\prime}\right)$ become Fréchet spaces. Moreover, the restriction mapping $C_{P}^{\mu, \infty}(\Omega) \rightarrow C_{Q}^{0, \infty}\left(\Omega^{\prime}\right)$ is closed and therefore continuous, by the closed graph theorem. Hence, to every integer $l>0$, there exists an integer $k>0$ and a constant $C>0$ such that

$$
w_{l}(f) \leqq C \max _{1 \leqq j \leqq k} v_{j}(f),
$$

for every $f \in C_{P}^{i, \infty}(\Omega)$. Applying condition (9) to the function

$$
f(x)=e^{i\langle x, \zeta\rangle},
$$

where $\zeta=\xi+i \eta$ and $\xi, \eta \in R^{n}$, we obtain the following lemma ${ }^{2}$.

LEMma 1. If the inclusion (8) holds then, for every integer $l>0$, we can find an integer $k>0$ and constants $C, c>0$ such that

2 We assume that $L^{\alpha}=D_{1}^{\alpha_{1}} D_{2}^{\alpha_{2}} \cdots D_{n}^{\alpha_{n}}$, where $D_{j}=-i\left(\partial / \partial x_{j}\right)$. 


$$
\left|Q^{l}(\zeta)\right| \leqq C\left(1+|\xi|^{\mu}\right)\left(1+\left|P^{k}(\zeta)\right|\right) e^{c|\eta|} \text {. }
$$

We denote by $N(P, a), V_{a}$ and $W_{a}$ the sets of all $\zeta=\xi+i \eta \in C^{n}$ such that $|P(\zeta) \leqq a,| \eta \mid \leqq a$ and $|\xi| \leqq a$, respectively.

LEMma 2. If condition (10) is satisfied, then $Q(\zeta)$ is bounded on every set $N(P, a) \cap V_{b}, a, b \geqq 0$.

Proof. Suppose there are $a, b \geqq 0$ such that $Q(\zeta)$ is not bounded on $N(P, a) \cap V_{b}$. Then the function

$$
s(t)=\sup _{\zeta \in N(P, a) \cap V_{b} \cap W_{t}}|Q(\zeta)|
$$

is defined and continuous for sufficiently large $t$, and

$$
s(t) \longrightarrow \infty \text { as } t \longrightarrow \infty \text {. }
$$

But, for a given $t, s(t)$ is the largest of all $s$ such that the equations and inequalities

$$
\begin{gathered}
|P(\xi+i \eta)|^{2} \leqq a^{2},|\eta|^{2} \leqq b^{2}, \\
|Q(\xi+i \eta)|^{2}=s^{2},|\xi|^{2} \leqq t^{2}, s \geqq 0, t \geqq 0,
\end{gathered}
$$

have a solution $\xi, \eta \in R^{n}$. Applying to (12) the Seidenberg-Tarski theorem and next a well-known argument (see [4], p. 276, or [6], p. 317 ) one shows easily that, for sufficiently large $t, s(t)$ is an algebraic function. We now expand $s(t)$ in a Puiseux series in a neighborhood of infinity and make use of (11). It follows that

$$
s(t)>t^{h}
$$

for some $h>0$ and all $t$ sufficiently large. On the other hand, $s(t)$ is assumed for some $\xi=\xi(t), \eta=\eta(t)$, and

$$
|\xi(t)| \leqq t
$$

Choosing in (10) $l>\mu h^{-1}$ we obtain a contradiction, which proves the lemma.

THEOREM 2. If $\mathscr{E}_{P}(\Omega) \subset \mathscr{E}_{Q}(\Omega)$, then the following equivalent conditions are satisfied:

$\left(\mathrm{I}_{1}\right) \quad Q(\zeta)$ is bounded on every set $N(P, a) \cap V_{b}$.

$\left(\mathrm{I}_{2}\right)$ For any $a \geqq 0$ there are constants $C, h>0$ such that

$$
|Q(\zeta)|^{h} \leqq C(1+|\eta|) \text {, for all } \zeta \in N(P, a) \text {. }
$$

$\left(\mathrm{I}_{3}\right)$ For any $b \geqq 0$ there are constants $C^{\prime}, h^{\prime}>0$ such that

$$
|Q(\zeta)|^{h^{\prime}} \leqq C^{\prime}(1+|P(\zeta)|), \text { for all } \zeta \in V_{b} \text {. }
$$


Proof. In view of Theorem 1, Lemma 1 and Lemma 2, we need only to show that conditions $\left(I_{1}\right)-\left(I_{3}\right)$ are equivalent. Also the implications $\left(I_{2}\right) \Rightarrow\left(I_{1}\right)$ and $\left(I_{3}\right) \Rightarrow\left(I_{1}\right)$ are obvious. We prove that $\left(I_{1}\right) \Rightarrow\left(I_{2}\right)$.

Consider the real polynomial

$$
\begin{aligned}
& W(\xi, \eta, r, s, t) \\
& \quad=\left(a^{2}-|P(\xi+i \eta)|^{2}-r^{2}\right)^{2}+\left(s^{2}-|\eta|^{2}\right)^{2}+\left(t^{2}-|Q(\xi+i \eta)|^{2}\right)^{2}
\end{aligned}
$$

of $2 n+3$ real variables. If $\xi, \eta \in R^{n}$ lie on the surface

$$
W(\xi, \eta, r, s, t)=0,
$$

then $\zeta=\xi+i \eta \in N(P, a)$. Moreover, by condition $\left(\mathrm{I}_{1}\right)$, the surface (13) is contained in a domain defined by an inequality

$$
|s|>\varphi(|t|),
$$

where $\varphi(\tau) \rightarrow \infty$ as $\tau \rightarrow \infty$. Applying now a theorem of Gorin ([1], Theorem 4.1) we conclude that there exist constants $C, h>0$ satisfying condition $\left(I_{2}\right)$. Thus $\left(I_{1}\right) \Rightarrow\left(I_{2}\right)$. The proof of the implication $\left(I_{1}\right) \Rightarrow\left(I_{3}\right)$ is similar.

3. Sufficient conditions. We now prove that conditions $\left(\mathrm{I}_{1}\right)-\left(\mathrm{I}_{3}\right)$ are sufficient for the inclusion under consideration. Our first goal is to construct a sequence of suitable fundamental solutions for the operators $P^{k}(D), k=1,2, \ldots$. We achieve this by modifying the construction of a fundamental solution for $P(D)$ given in [2].

In what follows $p$ and $q$ denote the orders of the differential operators $P(D)$ and $Q(D)$, respectively.

Lemma 3. Suppose that conditions $\left(\mathrm{I}_{1}\right)-\left(\mathrm{I}_{3}\right)$ are satisfied. Then there exist continuous functions $F_{k}, k=1,2, \cdots$, in $R^{n}$ with the following properties:

(a) For $\nu=p+q+n$ and any $k$,

$$
E_{k}=(\lambda-\Delta)^{\nu} F_{k}
$$

is a fundamental solution for $P^{k}(D)$, i.e.

$$
P^{k}(D) E_{k}=\delta
$$

(b) $P^{j}(D) F_{k}=F_{k-j}$, for $j=1,2, \cdots, k-1$.

(c) $Q^{l}(D) F_{k}, k, l=1,2, \cdots$, are continuous functions in $R^{n} \backslash\{0\}$.

(d) For any $l$ there is a $k$ such that $Q^{l}(D) F_{k}$ is a continuous function in $R^{n}$.

Proof. For any $\xi^{\prime}=\left(\xi_{1}, \cdots, \xi_{n-1}\right) \in R^{n-1}$, consider the subset of the complex $\zeta_{n}$-plane 


$$
U\left(\xi^{\prime}\right)=\left\{\zeta_{n} \in C:\left|P\left(\xi^{\prime}, \zeta_{n}\right)\right| \leqq 1 \text { or }\left.|\lambda+| \xi^{\prime}\right|^{2}+\zeta_{n}^{2} \mid \leqq 1\right\},
$$

where $\lambda>2 p$. There exist constants $C, h>0$ such that

$$
\left|Q\left(\xi^{\prime}, \zeta_{n}\right)\right| \leqq C\left(1+\left|\eta_{n}\right|^{h}\right),
$$

for all $\xi^{\prime} \in R^{n-1}$ and $\zeta_{n}=\xi_{n}+i \eta \in U\left(\xi^{\prime}\right)$. This follows from $\left(I_{2}\right)$, when $\left|P\left(\xi^{\prime}, \zeta_{n}\right)\right| \leqq 1$ and can be easily verified in the other case.

Let $U^{-}\left(\xi^{\prime}\right)$ be the union of all connected components of $U\left(\xi^{\prime}\right)$ having nonempty intersections with $C^{-}=\left\{\zeta_{n} \in C: \eta_{n}<0\right\}$. We denote by $L\left(\xi^{\prime}\right)$ the boundary of $C^{-} \cup U^{-}\left(\xi^{\prime}\right)$.

If $\zeta_{n} \in L\left(\xi^{\prime}\right)$, we have

$$
\left|P\left(\xi^{\prime}, \zeta_{n}\right)\right| \geqq 1 ;
$$

also there are constants $C^{\prime}, h^{\prime}>0$ (independent of $\xi^{\prime}$ ) such that

$$
\left|Q\left(\xi^{\prime}, \zeta_{n}\right)\right| \leqq C^{\prime}\left|P\left(\xi^{\prime}, \zeta_{n}\right)\right|^{h^{\prime}} \text {. }
$$

Inequality (16) is implied by $\left(I_{3}\right)$ and $(15)$, since $\left(\xi^{\prime}, \zeta_{n}\right) \in V_{2 p}$, when $\zeta_{n} \in L\left(\xi^{\prime}\right)$.

For $k=1,2, \cdots$, we set

$$
F_{k}(x)=\frac{1}{(2 \pi)^{n}} \int_{R^{n-1}}\left\{\int_{L\left(\xi^{\prime}\right)} \frac{e^{i\langle x, \zeta\rangle}}{\left(\lambda+\left|\xi^{\prime}\right|^{2}+\zeta_{n}^{2}\right)^{\nu} P^{k}(\zeta)} d \zeta_{n}\right\} d \xi^{\prime} .
$$

The functions $F_{k}$ are obviously continuous, because of (15). We claim that they satisfy the conditions (a)-(d).

Conditions (a) and (b) follow from general properties of the Fourier transforms of distributions.

The verification of condition (c) can be carried out in the same way as in [2] (see the proof of Lemma 4). We give a brief sketch of the argument.

Suppose first that, for a given $k, F_{k}^{(j)}$ is a function obtained by a construction as above, where the contour of integration (corresponding to $\left.L\left(\xi^{\prime}\right)\right)$ lies in the complex $\zeta_{j}$-plane; in particular $F_{k}^{(n)}=F_{k}$. Then

$$
Q^{l}(D)\left[F_{k}-F_{k}^{(j)}\right], j=1, \cdots, n-1 ; l=1,2, \cdots,
$$

are continuous functions in $R^{n}$; we omit the easy proof of this fact. Thus condition (c) will be verified, if we show that $Q^{l}(D) F_{k}^{(j)}, l=$ $1,2, \cdots$, are continuous for $x_{j} \neq 0(j=1, \cdots, n)$.

Consider, for example, the function $F_{k}$ and let $x_{n}<0$. In this case the contour $L\left(\xi^{\prime}\right)$ can be replaced by the boundary $V^{-}\left(\xi^{\prime}\right)$ of $U^{-}\left(\xi^{\prime}\right)$. By (14), there are positive constants $C_{1}$ and $C_{2}$ such that

$$
\eta_{n} \leqq-C_{1}\left|Q\left(\xi^{\prime}, \zeta_{n}\right)\right|^{1 / h}+C_{2}
$$

for all $\xi^{\prime} \in R^{n-1}$ and $\zeta_{n} \in V^{-}\left(\xi^{\prime}\right)$. Hence, if $\zeta=\left(\xi^{\prime}, \zeta_{n}\right)$, we have 


$$
\left|Q^{l}(\zeta) e^{i\langle x, \zeta\rangle}\right| \leqq|Q(\zeta)|^{l} \exp \left\{x_{n}\left(C_{1}|Q(\zeta)|^{1 / h}-C_{2}\right)\right\} .
$$

It follows that the integral

$$
\int_{R^{n-1}}\left\{\int_{V^{-\left(\xi^{\prime}\right)}} \frac{Q^{l}(\zeta) e^{i\langle x, r\rangle}}{\left(\lambda+\left|\xi^{\prime}\right|^{2}+\zeta_{n}^{2}\right)^{\nu} P^{k}(\zeta)} d \zeta_{n}\right\} d \xi^{\prime}
$$

converges absolutely and coincides with $Q^{l}(D) F_{k}(x)$, for every $l$.

In case $x_{n}>0$ we can reason similarly, replacing $L\left(\xi^{\prime}\right)$ by a contour $V^{+}\left(\xi^{\prime}\right)$ lying entirely in the half-plane $\eta_{n} \geqq 0$.

Condition (d) is a consequence of inequality (16). In fact,

$$
\frac{Q^{l}\left(\xi^{\prime}, \zeta_{n}\right)}{P^{k}\left(\xi^{\prime}, \zeta_{n}\right)}
$$

is bounded for $\xi^{\prime} \in R^{n-1}, \zeta_{n} \in L\left(\xi^{\prime}\right)$, whenever $k \geqq h^{\prime} l$.

Lemma 3 is now established.

THEOREM 3. If conditions $\left(\mathrm{I}_{1}\right)-\left(\mathrm{I}_{3}\right)$ are satisfied, the $\mathscr{E}_{P}(\Omega) \subset \mathscr{E}_{Q}(\Omega)$, for any open set $\Omega \subset R^{n}$.

Proof. Assume that $T \in \mathscr{C}_{P}(\Omega)$ and fix an arbitrary open set $\Omega^{\prime} \subset \subset \Omega$. We have to show that the restrictions of $Q^{l}(D) T, l=$ $0,1, \cdots$, to $\Omega^{\prime}$ are all in a space $\mathscr{D}^{\prime m}\left(\Omega^{\prime}\right)$.

By Lemma 3 , there are fundamental solutions $E_{k}$ for the operators $P^{k}(D), k=1,2, \cdots$, representable according to (a) with the functions $F_{k}$ satisfying conditions (b) - (d). Let $l$ be given and let $k$ be the integer corresponding to $l$ in condition $(\mathrm{d})$.

There are open sets $\Omega_{j}, j=0,1, \cdots, k+1$, such that

$$
\Omega^{\prime} \subset \subset \Omega_{k+1} \subset \subset \Omega_{k} \subset \subset \cdots \subset \subset \Omega_{0} \subset \subset \Omega .
$$

Since $T \in \mathscr{E}_{P}(\Omega)$, the restrictions of $P^{j}(D) T, j=0,1, \ldots$, to $\Omega_{0}$ are all of order $\leqq m_{0}$, say. For every $j=1,2, \cdots, k+1$, we now choose a function $\varphi_{j} \in \mathscr{D}\left(\Omega_{j-1}\right)$ such that $\varphi=1$ on $\Omega_{j}$. Then the distributions

$$
S_{1}=\varphi_{1} T, S_{j}=\varphi_{j} P(D) S_{j-1}, j=2,3, \cdots, k+1,
$$

are all of order $\leqq m_{0}$. Moreover

$$
S_{1}=T \text { on } \Omega_{1}
$$

and

$$
P(D) S_{j}-S_{j+1}=0 \text { on } \Omega_{j+1}, \quad j=1, \cdots, k .
$$

Making use of (a) we may write

$$
S_{1}=\sum_{j=1}^{k}\left[P(D) S_{j}-S_{j+1}\right] * E_{j}+S_{k+1} * E_{k},
$$


whence

$$
Q^{l}(D) S_{1}=\sum_{j=1}^{k}\left[P(D) S_{j}-S_{j+1}\right] * Q^{l}(D) E_{j}+S_{k+1} * Q^{l}(D) E_{k} ;
$$

here $*$ denotes the convolution. By (19), the "values" on $\Omega^{\prime}$ of each convolution

$$
\left[P(D) S_{j}-S_{j+1}\right] * Q^{l}(D) E_{j}
$$

depend on the values of $Q^{l}(D) E_{j}$ outside a neighborhood of the origin (see [5], Chapter VI, Theorem III). Therefore the restriction to $\Omega^{\prime}$ of the sum in (20) is a distribution of order $\leqq m_{0}+p+2 \nu$. On the other hand, the last term in (20) is of order $\leqq m_{0}+p+2 \nu$, because of (a) and (d). Hence the restriction of $Q^{l}(D) S_{1}$ to $\Omega^{\prime}$ is of order $\leqq m=m_{0}+p+2 \nu$ and $m_{0}$ can be chosen the same for all $l$. Since, by (18), the restrictions of $Q^{l}(D) S_{1}$ and $Q^{l}(D) T$ to $\Omega^{\prime}$ coincide, the theorem is proved.

Combining Theorem 2 with Theorem 3 we obtain the following corollary.

COROLLARY. Each of the conditions $\left(\mathrm{I}_{1}\right)-\left(\mathrm{I}_{3}\right)$ is necessary and sufficient for the inclusion $\mathscr{E}_{P}(\Omega) \subset \mathscr{C}_{Q}(\Omega)$, where $\Omega$ is any nonempty open set.

REMARK. Suppose that

$$
Q(\zeta)=P(\zeta) \sum_{j=1}^{n} \zeta_{j}^{2}
$$

where $P(\zeta)$ is an arbitrary polynomial. Then the operator $P(D)$ is $Q$-hypoelliptic (see [2], Theorem 1), but condition $\left(I_{3}\right)$ is not satisfied, unless $P(D)$ (and consequently $Q(D)$ ) is hypoelliptic.

\section{REFERENCES}

1. E. A. Gorin, Asymptotic properties of polynomials and algebraic functions of several variables, Uspehi Mat. Nauk, 16 (1961), 91-118.

2. E. A. Gorin and V. V. Grušin, Local theorems for partial differential equations with constant coefficients, Trudy Moskow. Mat. Obšč., 14 (1965), 200-210.

3. L. Hörmander, On the theory of general partial differential operators, Acta Math., 94 (1955), 161-248.

4. — Linear Partial Differential Operators, New York, 1969.

5. L. Schwartz, Théorie des Distributions I-II, Paris, 1957-1959.

6. F. Trèves, Linear Partial Differential Equations with Constant Coefficients, New York-London-Paris, 1966.

Received July 16, 1971.

SUNY-BUfFalo 



\section{PACIFIC JOURNAL OF MATHEMATICS}

\section{EDITORS}

\section{H. SAMElson}

Stanford University

Stanford, California 94305

C. R. Новву

University of Washington

Seattle, Washington 98105
J. DugunduI

Department of Mathematics University of Southern California

Los Angeles, California 90007

RICHARD ARENS

University of California

Los Angeles, California 90024

\section{ASSOCIATE EDITORS}
E. F. BECKENBACH
B. H. NeUmanN
F. WOLF
K. YosHIDA

\section{SUPPORTING INSTITUTIONS}

UNIVERSITY OF BRITISH COLUMBIA

UNIVERSITY OF SOUTHERN CALIFORNIA

CALIFORNIA INSTITUTE OF TECHNOLOGY

STANFORD UNIVERSITY

UNIVERSITY OF CALIFORNIA

UNIVERSITY OF TOKYO

MONTANA STATE UNIVERSITY

UNIVERSITY OF UTAH

UNIVERSITY OF NEVADA

WASHINGTON STATE UNIVERSITY

NEW MEXICO STATE UNIVERSITY

OREGON STATE UNIVERSITY

UNIVERSITY OF OREGON

OSAKA UNIVERSITY

UNIVERSITY OF WASHINGTON

* *

AMERICAN MATHEMATICAL SOCIETY

NAVAL WEAPONS CENTER 


\section{Pacific Journal of Mathematics}

\section{Vol. 43, No. $1 \quad$ March, 1972}

Alexander (Smbat) Abian, The use of mitotic ordinals in cardinal

arithmetic ....................................... 1

Helen Elizabeth. Adams, Filtrations and valuations on rings ......... 7

Benno Artmann, Geometric aspects of primary lattices .............. 15

Marilyn Breen, Determining a polytope by Radon partitions ........... 27

David S. Browder, Derived algebras in $L_{1}$ of a compact group .......... 39

Aiden A. Bruen, Unimbeddable nets of small deficiency .............. 51

Michael Howard Clapp and Raymond Frank Dickman, Unicoherent

compactifications ............................... 55

Heron S. Collins and Robert A. Fontenot, Approximate identities and the strict topology ................................... 63

R. J. Gazik, Convergence in spaces of subsets................. 81

Joan Geramita, Automorphisms on cylindrical semigroups ........... 93

Kenneth R. Goodearl, Distributing tensor product over direct product ..... 107

Julien O. Hennefeld, The non-conjugacy of certain algebras of

operators ................................... 111

C. Ward Henson, The nonstandard hulls of a uniform space ........... 115

M. Jeanette Huebener, Complementation in the lattice of regular

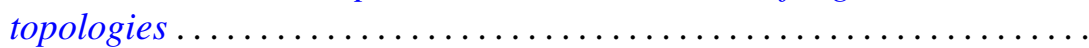

Dennis Lee Johnson, The diophantine problem $Y^{2}-X^{3}=A$ in a

polynomial ring .................................... 151

Albert Joseph Karam, Strong Lie ideals . . . . . . . . . . . . . . . . . . . . 157

Soon-Kyu Kim, On low dimensional minimal sets ............... 171

Thomas Latimer Kriete, III and Marvin Rosenblum, A Phragmén-Lindelöf

theorem with applications to $M(u, v)$ functions ..... . .

William A. Lampe, Notes on related structures of a universal algebra . . . . 189

Theodore Windle Palmer, The reducing ideal is a radical .

207

Kulumani M. Rangaswamy and N. Vanaja, Quasi projectives in abelian and module categories ................................ 221

Ghulam M. Shah, On the univalence of some analytic functions ......... 239

Joseph Earl Valentine and Stanley G. Wayment, Criteria for Banach

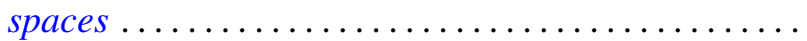

Jerry Eugene Vaughan, Linearly stratifiable spaces ............... 253

Zbigniew Zielezny, On spaces of distributions strongly regular with respect to partial differential operators ..................... 